\title{
The Need to Fabricate Lower Limb Prosthetic Devices by Additive Manufacturing
}

\author{
Fariborz Tavangarian* and Camila Proano
}

Mechanical Engineering Program, School of Science, Engineering and Technology, Pennsylvania State University, Harrisburg, Middletown $P A, 17057$, USA

*Corresponding author: Fariborz Tavangarian, Mechanical Engineering Program, School of Science, Engineering and Technology, Pennsylvania State University, Harrisburg, Middletown, PA, 17057, USA

\section{ARTICLE INFO}

Received: 幽 February 27, 2019

Published: March 13, 2019

Citation: Fariborz Tavangarian, Camila Proano. The Need to Fabricate Lower Limb Prosthetic Devices by Additive Manufacturing. Biomed J Sci \& Tech Res 15(5)-2019. BJSTR. MS.ID.002772.

\begin{abstract}
In this study, additive manufacturing is evaluated as a means of creating custom-made lower limb prosthetic devices. Amputees greatly benefit from having a custom-made prosthetic device. Furthermore, additive manufacturing has already proven successful in increasing the accessibility to custom-made hand prostheses, but there still needs to be an evaluation of how additive manufacturing could be employed in the creation of lower limb prostheses. This mini review encompasses a brief overview of the causes of lower limb amputations, the treatment plan for amputations, and some negative impacts of a poorly designed prosthesis. Additive manufacturing technologies as a potential fabrication method, their classifications, different materials that can be used, and an array of examples of how additive manufacturing has already been successful in medical fields will be discussed.
\end{abstract}

Keywords: Additive Manufacturing; Lower Limb Prosthesis; Polymers; 3D printing

\section{Introduction}

A prosthetic limb is an artificial replacement for a biological limb that may have been lost due to disease, injury, or deformation. For example, someone whose leg has been removed below the knee, a transtibial amputee, may choose to use a below-theknee prosthetic to help them regain some mobility [1]. With a prosthetic leg, he or she may be able to gain independence from a wheelchair. There are many styles of lower limb prostheses, such as running specific or the traditional design for simple walking. Each design provides its user with the movement they want and can physically handle. Figure 1 is an ottobock prosthetic leg made specifically for patients who want an active lifestyle. Around $60 \%$ of lower-limb amputees choose to use a prosthetic appendage, meaning that 30 million people worldwide require prosthetic limbs to carry out their daily activities [2]. In 2012, 1.9 million Americans were living with limb loss, a problem faced by all races. Non-whites constitute $42 \%$ of those living with limb loss [3]. In total, without including rehabilitation cost or prosthetic devices, America spends more than $\$ 8.3$ billion on the health care cost of amputations [4]. Throughout their lifetime, each American amputee will pay an average of $\$ 500,000$ in medical expenses.
$\$ 230,000$ alone can be attributed to the use of a single 5-year lower limb prosthetic device $[3,5]$.

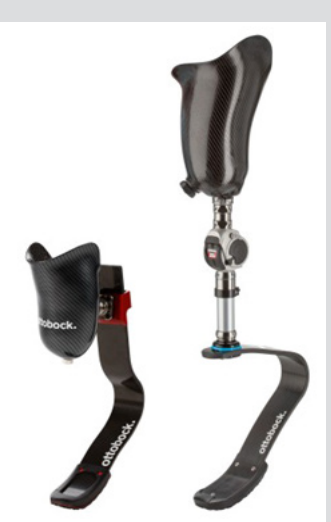

Figure 1: Running specific prostheses (Courtesy of Ottobock Co.)

Therefore, finding new techniques to lower the fabrication cost seems necessary. However, it is important to note that the medical expense of an amputation is not the only financial burden faced by amputees. Other financial costs may include the loss of 
employment opportunities, and work output [5]. 3D printing is one of the most promising approaches which can be used to alleviate the burden of medical expenses originated from amputations. Prosthetic limbs may help an amputee regain some of their mobility and independence. Although there are contributing factors that lead to amputations, people all around the world face the need of prosthetic limbs. Unfortunately, the cost of using a medical device after amputation may prevent some populations from accessing properly working devices. Therefore, it is important to consider the possibilities of using 3D printing as a method for increasing accessibility to prosthetic devices

\section{Causes of Lower Limb Amputations}

There are two primary sources of problems that lead to amputations. The number one cause of amputations is diabetes and its related ailments [6-8]. In 2008, 4 out of 1,000 adults with diabetes had a foot or leg amputation due to illnesses caused by diabetes [7]. The second leading reason for an amputation is traumatic injury, or a wound resulting from an external source [6-8]. Diabetes can damage the nerves of the foot and blood vessels. Additionally, it causes many secondary illnesses, such as peripheral arterial disease [6]. Diabetes patients have an increased risk of developing peripheral arterial disease if they are of advanced age, have had diabetes for a long time, or have uncontrolled hyperglycemia [9]. Some of the symptoms of peripheral arterial disease include cramping in the calf or buttocks muscles which worsens during walking [9]. Peripheral arterial disease, which affects 8-12 million Americans, hardens the patient's veins, restricting blood flow to limbs and eventually leading to amputations [7]. Due to the nerve damage, many sufferers of diabetes do not realize their foot has been injured, has ulcers, or is infected, thus wait too long to seek medical help [6-8].

The second leading cause of amputations is traumatic injury, accounting for 30,000 patients every year. Males between 15 and 30 years old are $80 \%$ more likely to be traumatic amputees than their female counterparts. The leading causes of amputations from traumatic injury are car accidents and injuries by machines [8]. Regardless of the reason for an amputation, the need for medical devices after amputations will constantly be present. Furthermore, as more cases of diabetes are diagnosed each year, it is important to investigate new methods for creating prosthetic devices.

\section{Prevention of Amputations}

Unfortunately, accidental amputations cannot always be prevented. Workplace injuries or traffic accidents may be inevitable, but their numbers can be reduced through rules and regulations. In the workplace, safety measures such as proper protective equipment, guardrails on equipment, and workplace rules can help prevent accidents leading to amputations. Accidents that happen outside of the workplace may be harder to avoid but may be reduced through education about what kinds of accidents may lead to an amputation. On the other hand, there are methods to limit planned amputations in diabetic patients. Planned amputations are a last resort for the advanced diabetic foot. They are necessary when there is too much necrotic tissue, or there is a life-threatening infection associated with the wounds [10]. There are treatments available to manage foot ulcers before they become severe enough to require amputation. For example, debridement removes dead tissue and stimulates the regrowth of healthy tissue by keeping a healthy granular wound bed $[9,11,12]$. For more severe injuries it is possible to do surgical debridement, which uses a scalpel to remove dead tissue and reveal a healthy skin base $[9,13]$. Other methods to clean out necrotic tissue include the use of once-daily topical agents, also known as enzymatic debridement. This method is not as drastic as surgery. Similarly, mechanical debridement can be achieved with a simple tool, but unfortunately can be painful as it can also remove healthy tissue. More recently, the use of maggots, known as biological debridement, has shown promising results to remove necrotic tissue [9]. In summary, the number of diabetic amputations could be reduced through early detection and proper treatment of ulcers.

There are safety measures which can be implemented to reduce situations which may lead to accidental amputations. Accordingly, the procedures used to prevent diabetic patients from losing a limb consist of cleaning the wounds to remove dead or infected tissue. Regrettably, not all amputations can be averted. Therefore, there are established methods of proving care for an amputee.

\section{Treatment of Transtibial Amputations}

Michigan Medicine at the University of Michigan explains that the healing process for a lower limb prosthesis has multiple stages to allow gradual healing and adjustment [14]. To be fitted for a prosthetic leg a patient must go through physical evaluations and an adjustment period to living without a prosthesis. In general, healthy patients who may have suffered an amputation due to an accident will be fitted for a customized lower limb prosthesis within 4 to 6 weeks after their amputation. On the other hand, amputations caused by diabetes may be able to have a prosthesis after a 6 to 8 -week period [14]. Furthermore, the process of how a patient is prepared to be fitted for a lower limb prosthesis is explained by the Brigham and Women's Hospital Department of Rehabilitation Services [15]. There are nine stages to an amputee's treatment plan [16].

Stage One: Preoperative: The patient is medically evaluated and educated about the procedure and the treatment process before the surgery takes place [15]. Additionally, the patient may begin a cardiopulmonary conditioning program $[15,16]$. The earlier the patient is evaluated and educated about the treatment process the better prepared the patient will be for the rehabilitation process $[15,17]$. 
Stage Two: Amputation Surgery/Dressing: After the surgical amputation steps are taken to ensure the patient will be able to wear a prosthetic device [15], the length of the residual limb is surgically measured, and the wound is closed. The residual limb is then reconstructed to maintain balance, and so it can support the stress caused by the prosthetic device $[15,17]$. With planned surgeries, it is important to try and keep the patient's knee with the purpose of reducing energy expenditure in the future $[15,18]$. The more limb that is removed, the more energy that prosthetic gait demands from the lungs and the heart $[15,19]$.

Stage Three: Acute Post-Surgical: This phase starts after the operation and ends once the patient is discharged from the hospital. The goal of this stage is to give the patients the tools for success before they can be outfitted for a prosthetic limb. Such devices include pain relief, workouts to increase muscle strength, phantom limb pain management, training on how to move without a prosthetic limb, and most importantly continued education and emotional counseling [15].

Stage Four: Pre-Prosthetic: This stage strictly prepares patients for the future use of a prosthetic device. During this phase of treatment, the patient undergoes residual limb shaping, stump shirking, skin care, and cardiovascular training [15]. Additionally, the patient works towards increasing their range of motion and continues to regain functional mobility without using a prosthesis [15]. The patient is also introduced to an Immediate Post-Operative Prosthesis which they will use to assimilate to the idea of wearing a prosthetic device $[14,15,20]$. This initial prosthesis is used while the limb is still healing and stabilizing [14].

Stage Five: Prosthetic Prescription/ Fabrication: To begin this stage, the wound must have healed and swelling reduced. Additionally, the stump must be mature and shaped like a cone $[15,21]$. Stage five aims to find a prosthetic device that will conform to the needs and the fitness condition of the patient [15]. It is interesting to note that if a patient weighs more than $330 \mathrm{lbs}$, it may be difficult to find a device which can accommodate the patient's weight $[15,22]$. Traditionally made prostheses are created using a casting of the residual limb with plaster and fiberglass bandages. Once the cast is dry, it is filled with a plaster slurry which will become a positive mold once the cast is destroyed [23]. The cast can then be used to create a mirror image of the stump, which will serve as the socket. The socket is latter attached to the rest of the prosthetic limb.

There are complications related to the defective fabrication of a prosthetic leg. Some of the problems associated with the construction of an inappropriate prosthesis may include [1]:

a) Vaulting, which happens when a prosthesis is too long and causes the patient to "step up" to complete a step,

b) Circumduction, in which the foot swings out while the leg is in motion, c) Lateral trunk bending, where the patient dips his or her shoulder on the side of the prosthesis while walking.

d) Foot slap, in which the foot moves when the heel makes contact with the ground.

e) Similarly, the foot being too soft can cause other problems such as drop-off, where the knee is suddenly flexed at the end of a step, and hyperextension of the knee on the affected side.

f) Finally, a loose-fitting socket can cause the individual to piston, or move the residual limb vertically while the weight shifts between legs.

Construction of a suitable prosthesis takes into consideration the patients input along with the knowledge and skills of the prosthetist. It provides stability, balance, and comfort both during walking and standing still. Most importantly, it minimizes the energy cost and contributes to gait symmetry. To keep walking as natural as possible the first contact between the ground and the prosthesis should be made with the heel of the foot, thus transferring the weight from the heel to the center of the foot, and finally the entire foot. To begin a step, the movement should start again with the heel and finish with the toes pushing off the ground [1].

Stage Six: Prosthetic Training: During this stage, the patient aims to increase the prosthesis' functionality and comfortable wear time. A patient will begin this stage ten or eleven weeks after their amputations. It is important to note that patients with a below-theknee amputation who use a soft dressing after amputation will have a temporary socket fabricated within six to eight weeks after their amputation surgery so they can be ready for this stage $[15,20]$.

Stage Seven: Community Integration: During this stage, the patient resumes all recreational activities to the best of their abilities. Some activities may require some modifications. Patients are encouraged to seek emotional support and continue using the coping strategies they were taught in the past stages [15].

Stage Eight: Vocational Rehabilitation: The patients in this step return to their line of work depending on the job requirements. Due to physical demands of previous jobs, some patients may choose to have a career change. The patient's return to work should be gradual and supplemented by professional counseling. Workload should increase over time, instead of being a sudden return to the amount of work that was appropriate before the amputation. Additionally, some training may be required to adapt to the person's new mobility [15].

Stage Nine: Follow Up: The final stage will continue for the rest of the person's life. This phase encompasses the lifelong use of a prosthetic device. Doctor visits should be scheduled every three months during the first 18 months, and twice a year after that $[15,16]$. An amputee is gradually prepared to comfortably wear their prosthesis for long periods of time. With a planned amputation, the patient starts preparing for the prosthetic device before the initial 
surgery takes place. The objective of the preparation is to help the patient return to a comfortable and functional life.

\section{Current Design for A Lower Limb Prosthesis}

When a patient loses their lower extremities, he or she may choose to use a prosthesis specific to their type of amputation. Lower limb prostheses are subdivided into categories depending on the height of the amputation. Hip disarticulations happen at the joint between the hip and the leg. Above-the-knee, amputations are called transfemoral amputations, while below-the-knee amputations are called transtibial amputations. Additionally, amputations at the ankle are called ankle disarticulations and finally, amputations at the foot are called partial foot amputations. As a reference, Figure 2 is a representation of the names and heights for lower limb amputations [2,24]. Each type of amputation requires different prosthetic devices. The parts of a below the knee prosthetic limb include the transtibial socket, the pylon, the tube adaptor, and the foot [2]. A prosthetic device for a transfemoral device is similar to one for a transtibial amputation, except that it includes an artificial knee. Figure 3 is a transfemoral prosthesis, with its socket, knee joint, pylon, ankle and foot labeled.

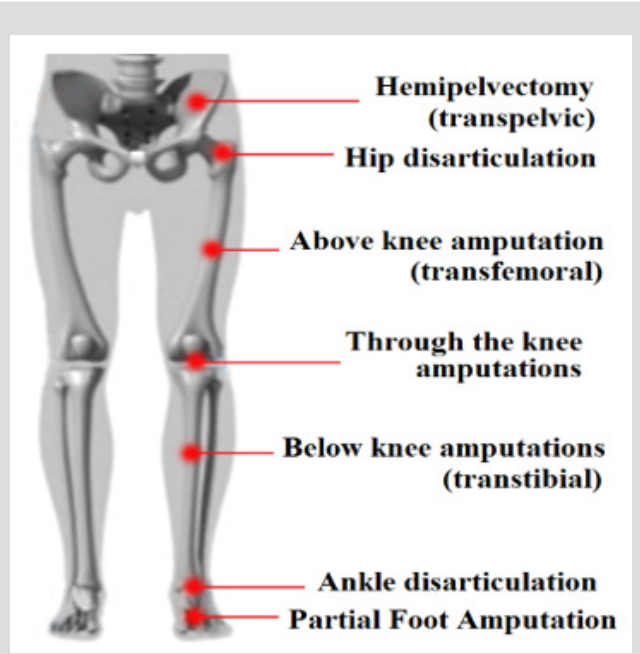

Figure 2: Names and heights for lower limb amputations. Adapted with permission from [24].

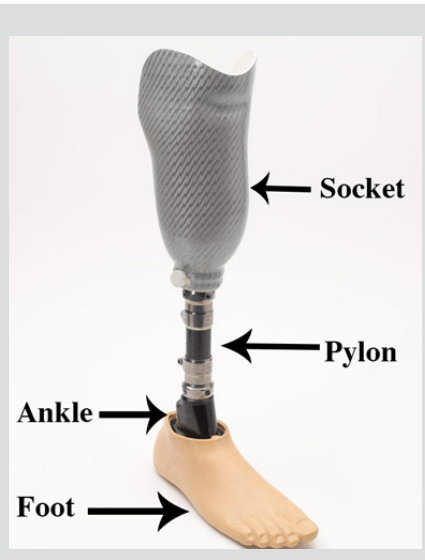

Figure 3: Labeled view of a lower limb prosthesis.
When looking at the prosthetic leg from top to bottom, the first section is the socket, which is designed to have properties similar to bone. The socket distributes the person's weight from the body to the ground. Therefore, conventional materials used for the socket are composites based on thermoset plastics mixed with other materials $[2,23]$. A socket is shaped like a cup to fit around the residual limb [23]. Furthermore, it is composed of three parts: the seating face, which connects the remaining limb to the prosthesis; the controlling socket area, which ensures correct motion of the leg; and the distal socket end, which transfers at most $10 \%$ of the person's weight [1]. Figure 4 is a visual representation of the subdivision of the socket. To keep a healthy stance, the socket must maintain the muscle gluteus medius stretched, and the pelvis balanced [1]. The socket facilitates the comfort and the functionality of the prosthesis [23]. The next section of the prosthetic limb is the pylon, which is described as a hollow aluminum pipe and serves as the connection between the socket and foot. Additionally, the pylon transfers the weight from the socket to the foot. Finally, the foot connects the prosthesis with the floor and provides energy absorption. The most economical designs for the foot are the solid ankle cushion heel and the stationary attachment flexible endoskeleton foot. Patients looking for increased mobility and stability may prefer an articulated single axis foot [2].

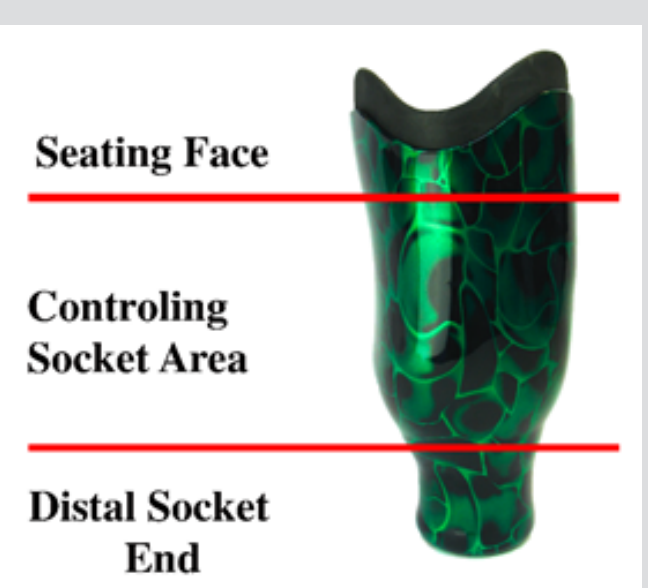

Figure 4: Hybrid polymer socket with labeled sections (Courtesy of 5280 Prosthetics LLC).

The current design for lower limb prosthesis mimics the limb it is replacing. In the case of a transtibial prosthetic device, the socket serves as the connection between the residual limb and the device. Similarly, the pylon is the equivalent of the tibia and fibula. Furthermore, the ankle and the foot in the prosthetic device mimic the design and purpose of their biological equivalents.

\section{Strength and Flexibility Requirements for Lower Limb Prostheses}

Each different category of lower limb prosthesis requires a different strength and flexibility limits. For example, a runningspecific prosthetic limb, such as a c-shaped prosthesis has different requirements than a prosthesis designed to provide its user with 
slow and steady walking. Rusu et al. [26] analyzed a computeraided model of a conventional C-shape ankle prosthesis with the use of Dynamic Designer Motion Professional module (DDMP) and tabulating motion values. A simplified model of the prosthesis was utilized to test the kinematic and dynamic parameters of the prosthesis in extended and flexed positions. The importance of this experiment was that the model could also be used for Finite Element Analysis, and thus with both tests, the maker could be sure the prosthesis would be reliable. The results showed that the forces excreted would not surpass the accepted values for the materials used [26]. Their study set a precedent for how future prosthetic limbs can be evaluated before production.

Running-specific prostheses must be evaluated for their flexibility. The 2017 study conducted by Oudenhoven et al. [27] explored the relationship between the preferred step frequency of athletes and the natural frequency of the running-specific prosthesis. They investigated the influence of the rate of steps on the stiffness of the leg. The motivation behind this experiment was to resolve the uncertainty surrounding past tests about stiffness regulation and running-specific prostheses. To collect data, they had participants run comfortably, hop, and jog on a treadmill and captured their velocities. The results showed that the athletes make their whole bodies stiffer to maximize the stiffness of the prosthetic limb when running. Furthermore, it was found that amputees can modify how fast they move their legs to keep up with the desired speed but do so much better with the uninjured leg. This experiment was significant because it indicated that stiffness in the runningspecific prostheses controls running performance [27].

Before a prosthetic device is used in patients, it is evaluated through computer simulations and physical testing. The characteristics of prosthetic devices affect their performance. There is an advantage from using computer simulations before physical testing takes place. If a computer model predicts that a design will fail physical testing, then adjustments can be made before the actual physical testing takes place. Furthermore, narrowing down designs through computer simulations simplifies the amount of material wasted during physical testing. Additionally, through testing, prosthetic devices can be redesigned for better performance.

\section{Lack of Accessibility to Prosthetic Specialist}

With regards to the importance of a properly fitting prosthetic device, it is important to note that not all populations have access to trained prosthetists. According to Kumar et al. [2], there is a worldwide deficit of 40,000 trained prosthetists. Nevertheless, 18,000 new professionals will join the field in the next half-century. Unfortunately, current prosthesis wearers do not have the luxury of waiting 50 years to work with a prosthetist on designing their custom-made prostheses. Studies have shown the importance of a custom-made prosthesis for the comfort and satisfaction of the user $[23,28,29]$. Custom-made prostheses often fit the patient better and will, therefore, have increased comfort and performance
[23]. Along with training more prosthetists, it may be important to find ways to make custom prosthetics devices more accessible to patients everywhere. One method for increasing the availability of personalized medicine is the use of additive manufacturing to create custom made items. Traditional custom-made prostheses are not always available in remote or underdeveloped locations. Therefore, additively manufactured prostheses pose a method for customization of medical devices by removing the need for laborintensive mold creations [23].

Additive manufacturing is defined as combining materials in a manner that, increases their efficiency, and diminishes the need for casts or templates [30]. To further personalize the products created through additive manufacturing, the design of medical devices is often aided by 3D imaging. Molds and templates are replaced by 3D models of the surface of scanned body parts, thus allowing for better fitting, and better working medical devices [30]. Such partnership between additive manufacturing and 3D imaging can be seen in the use of 3D scanners to create Invisalign dental aligners. Invisalign are clear aligners made of a thermoplastic material, which replaces traditional metallic braces. They allow dental patients to have a removable orthodontic treatment which can have the same effect as traditional metallic braces [31]. A good analogy for additive manufacturing is the structure of an onion. Each layer of an onion represents one layer of material laid down by the extruder head. All the layers combined make up the entire part, or in this example, the whole onion. Additive manufacturing allows for the creation of implants and prosthetics with intricate structural shapes made up of a mixture of materials [30]. Similarly, it allows for low-cost fabrication of medical devices in locations that may not have access to traditional manufacturing methods $[32,33]$.

The lower costs can be attributed to the reduced expenses in packaging, storage, and transportation of the desired products [32]. The process for creating a custom-made prosthesis with additive manufacturing has three steps: geometry capture, device design, and device manufacture [23]. Geometry capture can be completed with a foam box, a pin-based contact digitizer, optical molding, optical scanning, or through computer tomography and magnetic resonance imaging [23]. The computer-aided methods of capturing geometry will reduce the waste created during the creation of the fiberglass and plaster cast [23].

\section{Additive Manufacturing}

Additive manufacturing is an emerging field. The Wohler's Report explains that in 2015 the additive manufacturing market was worth \$5.1 Billion USD worldwide [32,34]. The market growth over the last 27 years could be partly attributed to the increased affordability of 3D printers. For example, in America, a do-ityourself kit for fused deposition modeling can be bought for 500 dollars and a printer for under $\$ 1,000$. A contributing factor to the price decrease in 3D printers is the expiration of essential patents for stereolithography, and selective laser sintering [32]. Additive 
manufacturing is already employed in the medical field. For example, in 2016, Van Galeen and her colleagues [35] experimented to create a new metal hip implant with built-in sensors to provide feedback on its condition. They ran into difficulties with the cassette design they included to monitor the hip implant once it was in the body. The design added unnecessary stress to the implant, which would ultimately contribute to its failure. Although there are still adjustments to be made to the prosthesis, the experiments proved that 3D printing the metal could be a gateway for faster testing and prototyping [35]. Table 1 provides some examples of areas where 3D printing is currently used in medicine [32].

Table 1: Subject areas where 3D printing is currently being used in medicine [32].

\begin{tabular}{|c|c|c|c|c|}
\hline Field & Drug Formulation & Modeling & Medical Devices & Tissues and Organs \\
\hline \multirow{3}{*}{ Application } & Controlled drug-delivery & Disease modeling for drug testing & Hearing aids & $\begin{array}{l}\text { Tissue analogues for } \\
\text { implantation }\end{array}$ \\
\hline & Personalized medicine & $\begin{array}{c}\text { Surgical planning, training and } \\
\text { education }\end{array}$ & $\begin{array}{l}\text { Implants, orthoses and } \\
\text { prostheses }\end{array}$ & \\
\hline & & Dental models & Surgical instruments and guides & \\
\hline
\end{tabular}

Additive Manufacturing Used for Prosthetic Devices: Additive manufacturing is not restricted to the manufacturing of implants; it can also be used for the production of prosthetic limbs such as hands. In a 2017 study conducted by Leddy et al. [36], a prosthetic finger made of composite material created using additive manufacturing, was compared to the prosthetic hands known as the Cyborg Beast, and the Bebionic v3. This experiment was conducted to validate the fabrication of composite prosthetic fingers as a viable option against current methods of production. The researchers propose a "middle of the road" option between very cheap 3D printed prostheses and professional prostheses. The hope was to influence future prosthetic designs, to create lightweight hand prosthetics that are durable, customizable, and cost-effective. Finally, by using carbon fiber, they were able to build prosthetic hands that are half as heavy as regularly printed 3D hand, and one-quarter of the weight of a machined aluminum hand with more flexible joints [36].

Acceptance of Prosthetics Like the Cyborg Beast in Developing Countries: A study evaluated the acceptance of 3D printed prosthetic devices in developing countries [5]. Patients in Port au Prince, Haiti, Amma, and Jordan were surveyed before and after they received 3D printed prosthetic hands which have proven to be a viable alternative to traditionally made prostheses. Figure 5 shows the offered hand prostheses to the patients in Haiti. The study revealed that the reception of 3D printed devices strongly depends on geographical location and people's beliefs. For example, patients in Haiti may have rejected the received prosthetic devices because of the strong cultural influences of the Voudu belief system. Voudu beliefs place great emphasis on an individual's spiritual wholeness, thus increasing the stigma of amputation. Similarly, patients in Jordan did not continue using the prosthetic devices given to them because many of the devices lack an anthropomorphic appearance.

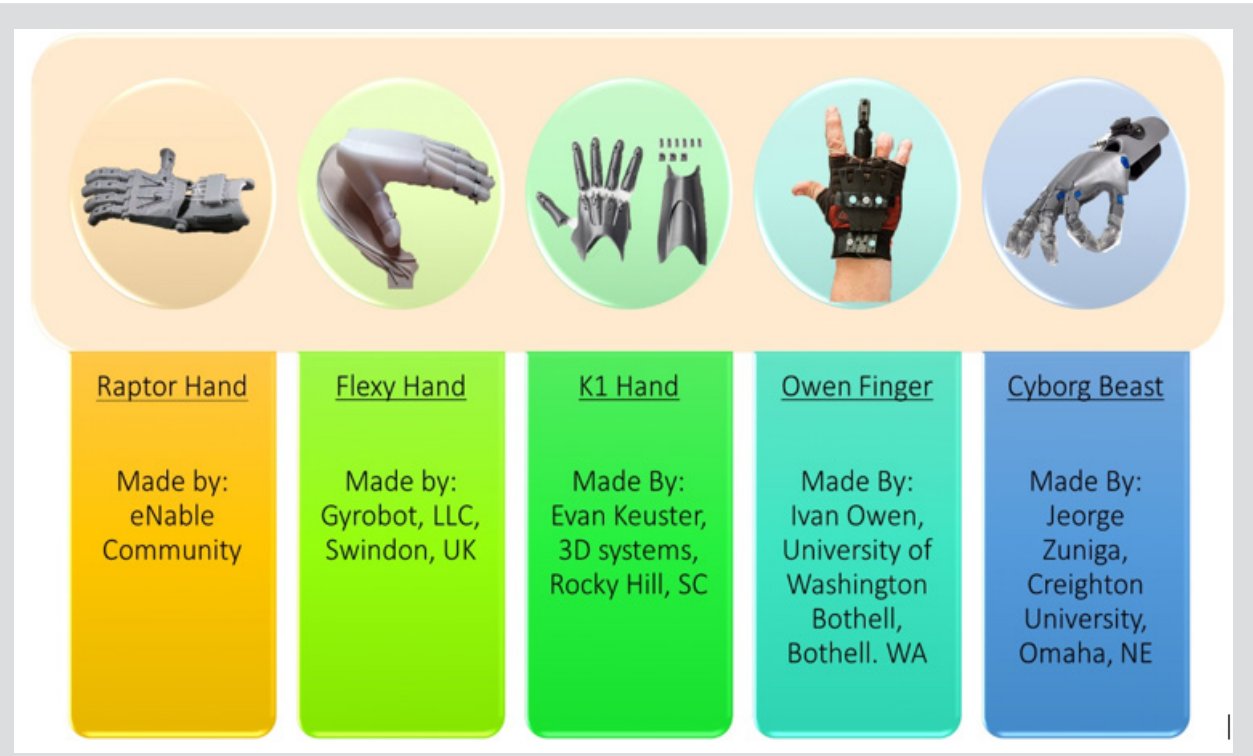

Figure 5: 3D printed hands used in acceptance study. 
The belief that "something is better than nothing" is not always true outside of western cultures. As demonstrated by Arabian's study there is a need for improving the appearance of 3D printed prosthetic devices to make them look as anthropometric and as natural as possible. While the efforts by nonprofit organizations like eNABLE and Jordanian Royal Medical Services to provide prosthetic devices to amputees should not be undermined, it is important for prosthetic designers to understand the cultural perception in individual markets [5]. The cultural acceptance of a 3D printed device will be different when it is presented to a patient in the United States, then when it is presented to a patient in the Middle Eastern region of the world or the Caribbean [5].

Types of Additive Manufacturing: To further understand the advantages of additive manufacturing it is important to outline the four main types of additive manufacturing technologies including vat-polymerization based printing, powder-based printing, droplet-based printing, and extrusion-based printing [32]. Each technology is further broken down to a particular kind of type of printing. During vat polymerization, a layer of photocurable resin is cured by a light of a distinct wavelength [32]. The desired piece is constructed one photocurable layer at a time. Furthermore, there are three technologies included under vat polymerization: stereolithography, digital light processing or continuous direct light processing, and liquid interface production. For further information about the different types of technologies, the readers are referred to [32]. Additionally, powder-based printing works by melting

Table 2: Classification of 3D printing technologies. powdered metals together through localized heating. Powderbased printing has four subcategories, selective laser sintering, electron beam melting, direct metal laser sintering, and selective laser melting. For further information please see $[32,37,38]$.

Similarly, droplet-based printing works by building up each layer of the material droplet by droplet [32]. The technologies which use droplet-based printing include multijet modeling, laser-induced forward transfer, and wax deposition modeling [32,39-41]. Finally, extrusion-based printing works by extruding a thermoplastic filament through a hot nozzle [32]. The technologies included in extrusion-based printing include direct ink writing and fused deposition modeling [34,37]. Fused deposition modeling is commonly seen in home 3D printing kits. It can be used to create small plastic parts that may not be available through other manufacturing methods. Table 2 shows the classifications of 3D printing technologies. One of the advantages of 3D printing is that products can be made with composite materials of preferred characteristics. A composite material is defined as a combination of materials that is not often found in nature. In most cases, their constituent phases are chemically different and have a clear separation from one another [41]. Additive manufacturing allows for the composition of the material amalgamation to be altered to produce the required material properties. For example, carbon can be added to plastics to increase the strength of the part, or titanium can be alloyed with aluminum to create a hard yet ductile metal mixture [30,42-44].

\begin{tabular}{|c|c|c|c|}
\hline Vat Polymerization Based & Powder Based & Droplet Based & Extrusion Based \\
\hline Stereolithography & Selective laser sintering & Multijet modeling & Fused deposition modeling \\
\hline Direct or indirect light processing & Direct metal laser sintering & Wax deposition modeling & Direct ink writing or Bio plotter \\
\hline $\begin{array}{c}\text { Continuous direct light processing } \\
\text { or Liquid interface production }\end{array}$ & Selective laser melting & Laser-induced forward transfer & $\begin{array}{c}\text { Pressure-assisted micro syringe or } \\
\text { Pressure-assisted printing }\end{array}$ \\
\hline & Electron beam melting & Binder jetting & \\
\hline
\end{tabular}

Stronger materials require larger forces to fail. Unfortunately, with increased strength also comes increased brittleness or little flexibility and resistance to impacts. On the other hand, ductile materials often lack high strength but will deform before breaking when exposed to large forces [45]. By producing composite materials, we can benefit from the desired properties of each component. Additionally, additive manufacturing allows greater control of the manufacturing processes, thus changing the material properties of parts. For example, a 2016 study conducted by Ting Guo et al. [46], demonstrated the correlation between printing parameters of poly (lactic-co-glycolic acid) and their effects on printing resolution. The study aimed to see how the inner diameter of the needle, the temperature of the material, the pressure, and the speed of printing affected 3D printed scaffolds that would be used for tissue engineering [46]. To determine the effects on printing the samples were evaluated with gel permeation chromatography to determine the molecular weight of the samples, and micro-computed tomography to characterize the samples as they were being printed.

They found that the high temperature of processing is not likely to break down the material as it is printing because a stable thermal energy is achieved. Additionally, they were able to see the relationship between needle size and the temperature needed to achieve an optimal viscosity of 0.1-10 Pa. s. Smaller needles require a lower viscosity. As the temperature of the printer increased, the viscosity of the material decreased [46]. Therefore, they concluded that high-temperature extrusion techniques would not harm the polymer during printing. Because melting temperature is mostly dependent on molecular weight, the lower printing temperatures were attributed to differences in molecular weights [46]. This finding may be relevant to future studies, as the printed scaffolds will have different mechanical and degradation properties than 
traditionally made structures. Due to the strict printing conditions of using a smaller needle, samples made with smaller needle sizes were more vulnerable to the material properties, than samples made with large needle sizes. On the other hand, in samples made with needles of large diameters, the fiber quality was affected by the pressure and speed of printing [46]. Plastics are not the only material which can be altered during 3D printing. There are other materials which can be used for additive manufacturing, such as metals, ceramics, and polymers. Summaries and examples of the kinds of materials that can be used for additive manufacturing are found in the following section.

Metals Used in Additive Manufacturing: There are several types of metals used for additive manufacturing. Stainless steel has two biocompatible alloys available for additive manufacturing, 304 and 316L [44]. Although the precious metals gold and platinum can also be processed through additive manufacturing, their high cost makes them unsuitable for mass production of medical devices [44]. Applications for other metals used in additive manufacturing are presented in the following section through summaries of experiments conducted with each material. Cobalt-chromium, titanium alloys, and stainless steel are commonly used in dental applications [47]. One study conducted by Alifui-Segbaya et al. [48] shows that additively manufactured cobalt chromium outperforms cast cobalt chromium in an environment with artificial saliva. Alifui-Segbaya and his team [48] aimed to see how the processing methods used in rapidly manufactured cobalt-chromium alloys would differ from the mechanical properties of cast materials. More specifically, they explored the corrosive effects of artificial saliva on a rapidly manufactured type 4 alloy, EOS SP2 (EOS GmbH, Electro Optical Systems, Germany), and a type 5 removable partial denture casting alloy, Vitallium casting alloy (Elephant Dental B.D., Netherlands).

They picked the two different alloys because they are very similar in chemical composition. For testing, the team followed the specifications under ISO 10271. Ten samples of each alloy were submerged in artificial saliva for 42 days. To compare the performance of each alloy, every few days readings were taken from an atomic absorption spectrometer to observe the amount of cobalt, chromium, and molybdenum ions released in parts per million [48]. Although Alifui-Segbaya and his team believe further research is necessary to mirror their findings, they discovered that the rapidly manufactured alloy showed lower levels of ion release than the cast material. They attributed the better performance of the type 4 alloy to its digitally controlled production method, which allowed the material to be very close to the manufacturer's specifications. On the other hand, the type 5 cast alloy's performance could be affected by the cooling times and melting temperatures that could not be controlled in a laboratory setting [48]. Similarly, commercially pure titanium and its alloys can be used to treat osseous defects. The properties of commercially pure titanium can be altered through its alloying elements, and through its manufacturing methods [44] For example, when used in the alloy Ti6Al4V, commercially pure titanium is a ductile material which properties can be altered to offer high fatigue resistance, good biocompatibility, and efficiency at promoting bone growth and healing [44].

Biocompatibility meaning the material will not harm living tissue. Additionally, high strength can be achieved in Ti6Al4V additively manufactured products because their formation allows the material to form a "martensitic-like structure" [44]. A martensite-like structure refers to the crystal structure in Ti6Al4V that resembles sharp needle-like branches in a core of the material. This crystal structure provides the material with very high strength, but also with very low ductility. The structure is formed by the rapid cooling of the Ti6Al4V as the printer deposits it. Therefore, the findings of the experiment demonstrate that titanium's strength can be increased by its manufacturing method and its ductility by the addition of alloying elements. According to Munsch [44], tantalum has similar characteristics to titanium. Therefore, it is currently used in the medical field for repairing bone defects. Stress shielding, or osteopenia, caused by the removal of normal pressure from the bone by an implant is a problem faced by patients with bone implants [49]. Porous implants made of materials such as Ti6Al4V can provide an artificial bone substitute material. The implant can promote bone ingrowth and reduction in stiffness for load-bearing applications. A study conducted by Wauthle et al. [50] used additive manufacturing to create a porous tantalum implant that simulates the structure and texture of human bones.

Wauthle and his research team [50] examined selective laser sintering as a manufacturing method to produce pure tantalum implants with desired mechanical properties and highly porous surfaces. Selective laser sintering is a type of 3D printing where a laser cures one layer of resin at a time. Then a platform lifts the cured layers out of the way, allowing a new uncured layer of resin is allowed to flow under the cured layer. The laser cures the newest layer of the resin, and the process continues until the entire part is created [32]. Static and dynamic fatigue testing was conducted on a specimen shaped like a rat' femur defect. It was concluded that selective laser sintering technology could be used to produce fine porous tantalum structures with a yield strength of $12.7 \mathrm{Mpa}$ and an elastic modulus of $1.22 \mathrm{Gpa}$, values within the range of human cancellous bone. The results showed $50 \%$ of bone ingrowth into the bone defect, meaning that the implant was osteoconductive. In conclusion, they proved that tantalum implants produced through selective laser sintering are a possible alternative to current methods of implant production [51]

Although magnesium and its alloys are biocompatible materials, they still need further research to overcome the restrictions caused by its material properties [44]. In 2015, Matena et al. [51] conducted a comparison between titanium and magnesium implants produced by selective laser melting method. Due to its release of gasses, magnesium and its alloys need a polymer coating to be safely used in bone grafting [51]. In this experiment, Matena's research team 
used a coating made of polycaprolactone (PLC) on the porous structure of bone implants produced by selective laser melting. The researchers aimed to provide an alternative to Ti6Al4V implants. Selective laser melting of magnesium and its alloys requires wetting solidified material with the liquid magnesium from the laser radiation. This process causes the surface of the parts to have no oxygen solubility, therefore exposing them to corrosion. The PLC coating could provide magnesium with increased corrosion resistance, therefore making it safe for bone tissue engineering applications [51]. Tissue engineering is defined as the use of 3D scaffolds with porous structures used to promote bone growth and regeneration. Additive manufacturing can provide a platform for combining metals or polymers in the production process.

Polymers Used for Additive Manufacturing: A polymer is made up of long repeating chains of organic elements, called monomers. Polymers are subdivided into two categories: thermosets and thermoplastics. Thermosets are those polymers that once they have been set through a chemical reaction will degrade when heated. Therefore, they are not suitable for additive manufacturing. On the other hand, thermoplastics are semi crystalline polymers which can be heated without degradation, allowing them to be manipulated during their cooling process. This behavior allows thermoplastics to be recycled [48]. Polyaryletherketones are a kind of thermoplastic polymers which have been used in orthopedic and spinal implants. They are a thermoplastic material with high levels of strength, temperature stability, and chemical resistance [44]. According to Munsch [44], polyamide is one of the most widely used synthetic materials used for selective laser sintering, a process in which polymer powders are heated and fused into the wanted 3D design [35]. The consolidation of polymers still needs further research to understand how more polymers could be used for additive manufacturing [47]. In an experiment conducted by Bai et al. [52], laser-sintered Polyamide 13 (PA12) was compared with a mixture of carbon nanotubes added to Polyamide 12 (PA12CNT). The carbon nanotubes were added with the hope of creating a material that could meet mechanical, thermal, and electrical requirements of specific applications.

The results of the experiment demonstrated that there was no significant change in the morphology of PA12 as compared to PA12-CNT. The near-spherical structure of both materials allows them to be continuously used for laser sintering. Similarly, carbon nanotubes were evenly spread throughout the PA 12 matrix. The balanced spreading of the carbon nanotubes ensures that the PA12CNT would have better properties than PA12. Therefore, the carbon nanotubes created more favorable conditions for laser sintering by increasing the thermal conductivity of PA12 by an average of $14.2 \%$. Furthermore, the shrinking of the material as it cools was reduced from $7.9 \%$ in PA12 to $3.7 \%$ in the PA12-CNT samples. Finally, PA12-CNT increased the tensile modulus of PA12 by $44.5 \%$ and the tensile strength by $7.0 \%$ [52]. Similarly, polycaprolactone is a biodegradable polymer that can also be used for selective laser sintering [44]. Mazzoli et al. [53] experimented in 2015 with polycaprolactone scaffolds manufactured through selective laser sintering for bone and cartilage repair. The polymer was printed into a cylindrical structure with interconnected holes that would allow cell growth. The study showed that PCL scaffolding created through selective laser sintering was a viable option for tissue engineering applications because it provided an environment that fostered cell growth [53].

Polylactides are also biocompatible materials and safely degrade within the body (bioresorbable) [44]. Some of the advantages of working with polylactides like polylactic acid (PLA) are that they can be made from renewable resources, and they have high strength and rigidity. Unfortunately, PLA is also brittle, meaning it has low flexibility and resistance to impacts [44]. In 2016, Chen et al. [30,54] conducted a study to strengthen PLA with carbon nanotubes introduced into the polymer through graphene oxide. The researchers investigated the printing of a composite made of thermoplastic polyurethane, polylactic acid, and graphene oxide, for its compatibility in tissue scaffolding. Specifically, they studied the mechanical and thermal properties and the effects of the printing orientation. For the mechanical properties, they conducted tensile and compression tests on printed cubes. To examine the consequences of printing direction, they performed tensile and compression tests on the other samples, which were placed in standing and lying positions. Finally, they tested to see how graphene oxide affects the growth of cells around the materials. Their method of 3D printing allowed them to improve thermal stability, understand the effects of printing orientation, and see how the addition of graphene oxide can affect cellular growth. This experiment furthers the understanding of what could be used for tissue engineering scaffolds [31].

Similarly, a study discussed earlier by Tin Guo et al. [46] also aimed to understand the effectiveness of a 3D printed scaffold for tissue engineering. A correlation between the presence of methyl side groups in lactic acid and the rate of water absorption in the material was noted. The scaffolds with a lower ratio of lactic acid to glycolic acid had a greater water absorption capability [46]. Similarly, the presence of an acid end cap in the molecular structure of the material decreased the hydrophobicity and accelerated the degradation of the material [46]. Therefore, poly (lactic-co-glycol acid) with a high ratio of lactic acid to glycolic acid combined with an ester end cap can be used to create 3D printed scaffolds suitable for cartilage regeneration because it has mechanical properties similar to human cartilage and a slow rate of degradation [46].

Strengthening of Polymers Through Carbon: As demonstrated by the studies summarized above, polymer materials can be reinforced through the addition of carbon nanotubes $[30,44,46]$. Many studies have been conducted to test the validity of polymer strengthening through carbon nanotubes [30,55]. The experiment conducted by Xinhua et al. [54] in 2016 supplements 
the studies mentioned above by evaluating the impact of carbon fiber embedded in 3D printed structures. The study evaluated structures made with polylactic acid and continuous carbon fibers. The influence of carbon fibers on the mechanical properties of 3D printed structures as well as the biodegradability of the material was evaluated. Without reinforcements, 3D printed structures made of polymers are sometimes too weak for certain applications. Polyacrylonitrile with continuous carbon fibers mixed with E-44 epoxy resin and a low molecular polyamide adhesive was used to create the testing samples. Samples were fabricated by printing one layer of the polymer and using the adhesive and the resin to bind the carbon fibers to the printed layer.

Uniaxial tension tests and three-point bending tests were conducted to evaluate the strength and strain of the specimens. They found that adding carbon fibers to the structure of polyacrylonitrile increased their strength. These findings were in good agreement with previous studies that showed carbon fibers were a good indicator of when the polymer had been tested past its yield point [54]. Finally, the researchers evaluated their results with the real-world application of a $3 \mathrm{D}$ printed prosthetic hand [54]. Similarly, a study was conducted to assess the effectiveness of welding 3D printed carbon nanotube polymer composites by applying heat directly at the point of welding between the nanotube and the surface of the 3D printed polymer [55]. In the past, the carbon filament was bonded to the 3D printed structure by heating the entire polymer past its critical sintering temperature [55]. The process created a problem because uniform heating of the 3D printing structure would cause the structure to deform, and lack of heat creates a weak bond between the carbon nanotube and the polymer [55]. A trouser tear test was performed to check the strength of the weld between the nanotube and the polymer. The test allowed the researchers to estimate the fracture strength of a 3D printed layer weld line when it is pulled in two opposing horizontal directions. The fracture strength of the welded parts increased by $275 \%$ as compared with a regularly printed part [55]. Additionally, the welded parts were not deformed by the welding process. Therefore, the welding process effectively increases the bonds between printed layers without distorting the shape of the parts [55].

In summary, there have been in-vitro advantages to using additively manufactured products were traditionally made products were used. The benefits come from the ability to manipulate the constituents of the material, and the increased manufacturing controls that are available with additive manufacturing.

\section{Conclusion}

In conclusion, there is an ever-present need to provide amputees with custom-made prosthetic devices. In some regions of the world, there is a lack of accessibility to trained professionals who could provide patients with quality custom-made prosthetic devices. The need for increased accessibility to hand prostheses is currently being addressed through additively manufacturing prosthetic hands and finger. Therefore, it is important to evaluate the possibility of using the materials found in an at home 3D printer kit to create an acceptable lower limb prosthesis. Furthermore, the advantage of using additive manufacturing to create medical devices previously made by traditional methods such as casting show promising results in the area of prosthetics. Such benefits include the increased control over material composition and manufacturing techniques. There is still further evaluation needed with regards to the use of 3D printed lower limb prosthetics. It is hoped that removing the need for labor-intensive mold creation for lower limb prostheses can be bypassed through the use of computer models and 3D scanning combined with 3D printing. Therefore, creating custom-made lower limb prosthetic devices available to a wider audience.

\section{References}

1. Pitkin MR (2010) Biomechanics of Lower Limb Prosthetics (1 $1^{\text {st }}$ edn.), Springer-Verlag Berlin Heidelberg Berlin Heidelberg.

2. Kumar PK, Charan M, Kanagaraj S (2017) Trends and Challenges in Lower Limb Prosthesis. IEEE Potentials 36(1): 19-23.

3. https://www.amputee-coalition.org/wp-content/uploads/2014/09/ lsp_Roadmap-for-Limb-Loss-Prevention-and-Amputee-CareImprovement_241014-092312.pdf

4. https://www.cdc.gov/ncbddd/disabilityandhealth/features/limb-lossawareness.html\#ref3

5. Arabian A, Varotsis D, McDonnell C, Meeks E (2016) Global social acceptance of prosthetic devices in 2016 IEEE Global Humanitarian Technology Conference (GHTC) 563-568.

6. https://www.cdc.gov/chronicdisease/resources/publications/aag/ pdf/2016/diabetes-aag.pdfawareness.html\#ref3

7. http://www.webmd.com

8. Sheehan TP, Gondo GC (2014) Impact of limb loss in the United States. Physical medicine and rehabilitation clinics of North America 25(1): 9-28.

9. Amin N, Doupis J (2016) Diabetic foot disease: From the evaluation of the "foot at risk" to the novel diabetic ulcer treatment modalities. World Journal of Diabetes 7(7): 153-164.

10. http://www.mayoclinic.org/diseases-conditions/diabetes/in-depth/ amputation-and-diabetes/ART-20048262?pg=1.

11. Falabella AF (2006) Debridement and wound bed preparation. Dermatologic Therapy 19(6): 317-325.

12. Veves A, Giurini JM, LoGerfo FW, SpringerLink (2012) The Diabetic Foot: Medical and Surgical Management 3rd 2012. 3 Aufl 3 ed Humana Press Totowa NJ.

13. Steed DL, Donohoe D, Webster MW, Lindsley L (1996) Effect of extensive debridement and treatment on the healing of diabetic foot ulcers. Diabetic Ulcer Study Group. Journal of the American College of Surgeons 183(1):61-4.

14. http://www.uofmhealth.org/conditions-treatments/lower-extremityamputation.

15. https://www.brighamandwomens.org/assets/BWH/patients-andfamilies/pdfs/general---le-amputation.pdf.

16. Srivastava K, Chaudhury S (2014) Rehabilitation after amputation: psychotherapeutic intervention module in Indian scenario. Journal of the American Podiatric Medical Association 1-6. 
17. Esquenazi A (2004) Amputation rehabilitation and prosthetic restoration. From surgery to community reintegration. Disability and rehabilitation 26(14-15): 831-836

18. Aulivola B, Hile CN, Hamdan AD, Sheahan MG, Veraldi JR, et al. (2004) Major Lower Extremity Amputation: Outcome of a Modern Series. Archives of Surgery 139(4): 395-399.

19. Cutson TM, Bongiorni D, Michael JW, Kochersberger G (1994) Early Management of Elderly Dysvascular Below-Knee Amputees. JPO Journal of Prosthetics and Orthotics 6(3): 62-66.

20. Seymour RPD (2002) Prosthetics and orthotics: lower limb and spinal Lippincott Williams \& Wilkins Philadelphia.

21. Murray CD, Pettifer S, Howard T, Patchick EL, Caillette F, et al. (2007) The treatment of phantom limb pain using immersive virtual reality: three case studies. Disability and rehabilitation 29(18): 1465-1459.

22. Cole ES (2003) Training elders with transfemoral amputations. Topics in Geriatric Rehabilitation 19(3): 183-190.

23. Jin Ya, Plott J, Chen R, Wensman J, Shih A, et al. (2015) Additive Manufacturing of Custom Orthoses and Prostheses -A Review. Procedia CIRP 36:199-204.

24. Kalantarov S, Riemer R, Oron Gilad T (2018) Pedestrians road crossing decisions and body parts' movements. Transportation Research Part F: Traffic Psychology and Behaviour 53: 155-171.

25. Rajtúková V, Michalíková M, Bednarčíková L, Balogová A, Živčák J, et al. (2014) Biomechanics of Lower Limb Prostheses. Procedia Engineering 96: 382-391.

26. Rusu L, Toth Tascau M, Toader Pasti C (2013) Dynamic Analysis of a Lower Limb Prosthesis. Applied Mechanics and Materials 430: 230-234.

27. Oudenhoven LM, Boes JM, Hak L, Faber GS, Houdijk H, et al. (2017) Regulation of step frequency in transtibial amputee endurance athletes using a running-specific prosthesis. Journal of Biomechanics 51: 42-48.

28. Berke GM, Fergason J, Milani JR, Hattingh J, McDowell M, et al. (2010) Comparison of satisfaction with current prosthetic care in veterans and servicemembers from Vietnam and OIF/OEF conflicts with major traumatic limb loss. Journal of rehabilitation research and development 47(4): 361-371.

29. Gailey R, Allen K, Castles J, Kucharik J, Roeder M, et al. (2008) Review of secondary physical conditions associated with lower-limb amputation and long-term prosthesis use. Journal of rehabilitation research and development 45(1): 15-29.

30. Chen Q, Mangadlao JD, Wallat J, De Leon A, Pokorski JK, et al. (2017) 3D Printing Biocompatible Polyurethane/Poly (lactic acid)/Graphene Oxide Nanocomposites: Anisotropic Properties. ACS applied materials \& interfaces 9(4): 4015-4023.

31. https://www.invisalign.com

32. Liaw CY, Guvendiren M (2017) Current and emerging applications of 3D printing in medicine. Biofabrication 9(2): 024102.

33. Rankin TM, Giovinco NA, Cucher DJ, Watts G, Hurwitz B, et al. (2014) Three-dimensional printing surgical instruments: are we there yet? The Journal of surgical research 189(2): 193-197.

34. http://wohlersassociates.com/press71.htm

35. Van Gaalen JB, Trejos AL, Nikolov HN, Ivanov TG, Pollman SI, et al. (2016) Versatile smart hip implant technology using 3D metal printing. In IEEE pp. 2731-2734.

36. Leddy MT, Belter JT, Gemmell KD, Dollar AM (2015) Lightweight custom composite prosthetic components using an additive manufacturingbased molding technique. in IEEE pp. 4797-4802.
37. Wong KV, Hernandez A (2012) A Review of Additive Manufacturing. ISRN Mechanical Engineering. 1-10.

38. Shirazi SFS, Gharehkhani S, Mehrali M, Yarmand H, Metselaar HSC, et al. (2015) A review on powder-based additive manufacturing for tissue engineering: selective laser sintering and inkjet 3D printing. Science and Technology of Advanced Materials 16(3): 33502.

39. Upcraft S, Fletcher R (2003) The rapid prototyping technologies. Assembly Automation 23(4): 318-330.

40. Do AV, Khorsand B, Geary SM, Salem AK (2015) 3D Printing of Scaffolds for Tissue Regeneration Applications. Advanced Healthcare Materials 4(12): 1742-1762.

41. Ozbolat IT (2015) Scaffold-Based or Scaffold-Free Bioprinting: Competing or Complementing Approaches? Journal of Nanotechnology in Engineering and Medicine 6(2): 024701.

42. Lewis JA (2006) Direct Ink Writing of 3D Functional Materials. Advanced Functional Materials 16(17): 2193-2204.

43. Callister WD, Rethwisch DG (2014) Materials science and engineering: an introduction 9th ed John Wiley and Sons. Inc Hoboken NJ.

44. Brandt M (2016) Laser Additive Manufacturing: Materials Design Technologies and Applications Elsevier Science Kent pp. 498.

45. Budynas RG, Nisbett JK, Shigley JE (2011) Shigley's mechanical engineering design 9 th ed. McGraw-Hill New York.

46. Guo T, Holzberg TR, Lim CG, Gao F, Gargava A, et al. (2017) 3D printing PLGA: a quantitative examination of the effects of polymer composition and printing parameters on print resolution. Biofabrication 9(2):024101.

47. Mehboob H, Chang S-H (2014) Application of composites to orthopedic prostheses for effective bone healing: A review. Composite Structures 118: $328-341$

48. Alifui Segbaya F, Foley P, Williams RJ (2013) The corrosive effects of artificial saliva on cast and rapid manufacture-produced cobalt chromium alloys. Rapid Prototyping Journal 19: 95-99.

49. Niinomi M, Nakai M (2011) Titanium-Based Biomaterials for Preventing Stress Shielding between Implant Devices and Bone. International Journal of Biomaterials: 1-10.

50. Wauthle R, Van Der Stok J, Yavari SA, Van Humbeeck J, Kruth JP, et al. (2015) Additively manufactured porous tantalum implants. Acta Biomaterialia 14: 217-225.

51. Matena J, Petersen S, Gieseke M, Teske M, Beyerbach M, et al. (2015) Comparison of Selective Laser Melted Titanium and Magnesium Implants Coated with PCL. International journal of molecular sciences 16(6): 13287-13301

52. Bai J, Goodridge RD, Hague RJ, Song M (2012) Carbon nanotube reinforced Polyamide 12 nanocomposites for laser sintering. 23rd Annual International Solid Freeform Fabrication Symposium - An Additive Manufacturing Conference pp. 98-107.

53. Alida M, Ferretti C, Gigante A, Salvolini E, Mattioli-Belmonte M, et al. (2015) Selective laser sintering manufacturing of polycaprolactone bone scaffolds for applications in bone tissue engineering. Rapid Prototyping Journal 21(4): 386-392.

54. Yao X, Luan C, Zhang D, Lan L, Fu J, et al. (2017) Evaluation of carbon fiber-embedded 3D printed structures for strengthening and structuralhealth monitoring. Materials \& Design 114(15): 424-432.

55. Sweeney CB, Lackey BA, Pospisil MJ, Achee TC, Hicks VK, et al. (2017) Welding of 3D-printed carbon nanotube-polymer composites by locally induced microwave heating. Science Advances 3. 


\section{ISSN: 2574-1241}

DOI: 10.26717/BJSTR.2019.15.002772

Fariborz Tavangarian. Biomed J Sci \& Tech Res

(c) $(7)$ This work is licensed under Creative

Submission Link: https://biomedres.us/submit-manuscript.php

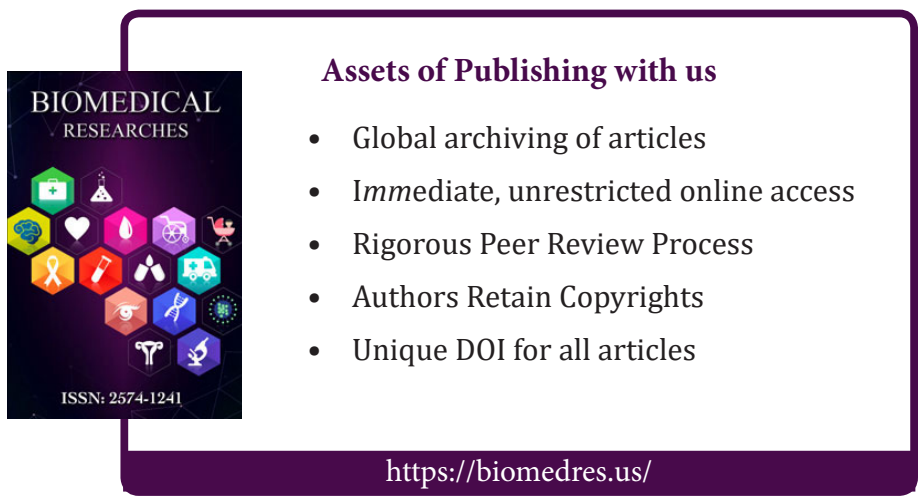

